\title{
Factores de riesgo que influyen en la retención urinaria en los pacientes posoperados inmediatos de la Clínica Good Hope, Lima
}

\author{
Dámaris Idrogo-Sánchez ${ }^{1 *}$, Brenda Peña-Jiménez¹, Bertha Alcócer-Rojas² \\ ${ }^{I}$ Clínica Good Hope - Malecón Balta 956, Miraflores - Lima, Perú \\ ${ }^{2}$ Hospital Jorge Voto Bernales Corpancho - Km 3.5, Carretera Central-Santa Anita
}

\begin{abstract}
RESUMEN
Objetivo: Identificar los factores de riesgo que influyen en la retención urinaria de los pacientes posoperados inmediatos de la Clínica Good Hope, abril 2012. Material y Métodos: El estudio fue de tipo cuantitativo, descriptivo, transversal, de diseño de casos y controles. Conformado por 85 pacientes posoperados inmediatos. Se aplicó una guía de recolección de datos con la finalidad de conocer los factores de riesgo asociados con la retención urinaria. Se realizó el análisis a través de Odds Ratio, (OR) de productos cruzados con su respectivo intervalo de confianza (IC) y significancia al 95\%. Resultados: El 51,8\% de la muestra pertenece al sexo femenino; más de la mitad (58,8\%) tienen más de 40 años. Los posoperados obtuvieron valores que se interpretan como posibilidades de presentar retención urinaria: los posoperados con antecedentes de adenoma de próstata 11,6 veces razones de riesgo; los intervenidos por traumatología presentaron 4,4 veces razones de riesgo y los sometidos bajo anestesia raquídea tuvieron 11 veces razones de riesgo. Conclusiones: Se identificaron 9 factores de riesgo de los cuales 3 de ellos obtuvieron mayor razón de ventaja de presentar retención urinaria en el posoperatorio inmediato, concluyendo que los pacientes intervenidos bajo anestesia raquídea tienen mayor probabilidad de desarrollar retención urinaria en el posoperatorio inmediato.
\end{abstract}

Palabras clave: Factor de riesgo, retención urinaria, post operado inmediato.

\begin{abstract}
Objective: Identify the risk factors influencing urinary retention in patients post operated immediate clinical good Hope, April 2012. Material and Methods: The study was quantitative, descriptive, transversal, not experimental, type of design cases and controls. comprised of 85 patients immediate posoperados. A guide to data collection with the purpose of knowing the risk factors associated in urinary retention was applied. The analysis was performed through Odds Ratio (OR) of cross products with its respective confidence interval (CI) and significance at 95\%. Results: $51.8 \%$ Of the sample belonging to the female sex; more than half (58.8\%) have more than 40 years. The postoperados values are interpreted as possibilities of presenting urinary retention were: the posoperados with a history of 11.6 times prostate adenoma risk reasons; seized by Traumatology presented 4.4 times risk reasons and the subject under spinal anesthesia had 11 times risk reasons. Conclusions: 9 Risk factors were identified, of which 3 of them obtained more reason of advantage of presenting urinary retention in the immediate postoperative concluding that patients operated on under spinal anesthesia are more likely to develop urinary retention in the immediate postoperative.
\end{abstract}

Keywords: Factor risk, urinary retention, immediate operated post. 


\section{INTRODUCCIÓN}

La atención posoperatoria comienza cuando concluye la intervención quirúrgica. La primera fase se caracteriza como recuperación inicial, en la que se presentan cambios en los diferentes órganos y sistemas por efecto de la anestesia, denominada así la fase posoperatoria inmediata. La recuperación de la anestesia supone un proceso gradual de funciones orgánicas y reflejos vitales que pueden asociarse a importantes adversidades con graves consecuencias para la vida del paciente. Por tal motivo, los pacientes anestesiados son monitorizados de modo permanente en la Unidad de Recuperación Posanestésica, según el Manual de Enfermería (Océano/Centrum, 1997).

Morgan, Murray, \& Mikhail (2003) hacen referencia que, al someter a un paciente a cualquier práctica quirúrgica implica riesgos derivados de la anestesia o de la cirugía. Aquellas complicaciones o riesgos anestésicos más comunes en bloqueos regionales ya han sido identificados en pacientes sometidos a cirugía general. Entre este conjunto de complicaciones más frecuentes, derivadas de la anestesia regional, están la hipotensión ortostática inducida por la anestesia, bloqueo motor o sensitivo prolongada, cefalea y retención urinaria.

La retención urinaria se conoce también como bloqueo de orina y esta tiene lugar cuando existe un aumento de liberación de catecolaminas. Se cree que los receptores alfa adrenérgicos del músculo liso del cuello vesical y la uretra son estimulados por estas hormonas, aumentando su tono por este mecanismo, produciendo retención urinaria. (Smeltzer \& Bare, 2005).

Barretto de Carvalho, Vieira da Costa, \& Saraiva (2007) realizaron un estudio con el objetivo de determinar la incidencia de retención urinaria posoperatoria en pacientes que se encontraban en uso de analgésicos opioides, así como describir el método utilizado en el vaciado vesical. De ellos, 594 pacientes no usaron cateterismo de demora en el preoperatorio. Asimismo, 128 pacientes de este grupo presentó retención urinaria, con una incidencia del 22\% (128/594). Hubo una asociación estadísticamente significativa entre la ocurrencia de retención urinaria y el uso de analgesia epidural continua $(\mathrm{p}=0,009)$. El 69\% de los pacientes presentó una micción espontánea, luego de haber realizado apenas un cateterismo.

El número de casos de retención urinaria no es de gran porcentaje, como se puede apreciar. Aparentemente no es una cifra alarmante o preocupante; sin embargo, no ha sido considerado objeto de estudio para hacer un seguimiento, con la finalidad de detectar las causas y/o factores de riesgo que conllevan a esta complicación posoperatoria, considerando que en toda institución donde se efectúan cirugías de complejidad, siempre habrá un mínimo porcentaje de incidencia de retención urinaria. (Hamlin, 2010).

Algunos autores como (Smeltzer \& Bare, 2005), (Long, 1998) y (Chocarro \& Venturini, 2005) entre otros, identificaron que entre los factores de riesgo desencadenantes de una retención urinaria están el género, edad, antecedentes patológicos asociados a vías urinarias, tipo de cirugía, tiempo operatorio, tipo de anestesia, dosis utilizada de narcóticos y la cantidad de volumen transfundido.

En la Clínica Good Hope, el tiempo de permanencia en la URPA es de 2 horas como mínimo y un máximo de 4 horas, tiempo establecido por la jefatura del servicio, posterior a este período el paciente debe estar apto para ser trasladado a su habitación. En este lapso de tiempo el paciente debe haber recuperado sus funciones motoras y sensoriales, estar orientado, sus signos vitales estables y que no haya evidencia de hemorragia $\mathrm{u}$ otras complicaciones.

En este sentido se planteó como objetivo de investigación, identificar los factores de riesgo que influyen en la retención urinaria en los pacientes postoperados inmediatos de la Clínica Good Hope, abril 2012

\section{MATERIAL Y MÉTODOS}

El presente estudio se realizó en la Clínica Good Hope, ubicado en el distrito de Miraflores. La población estuvo constituida por 85 pacientes posoperados inmediatos sometidos a cirugía durante el mes de abril del 2012.

Se incluyeron el $100 \%$ de los pacientes posoperados inmediatos, teniendo en consideración criterios de inclusión: pacientes postoperados sometidos bajo anestesia general, epidural o raquídea y pacientes de edades a partir de los 18 años en adelante. Entre los criterios de exclusión, fueron aquellos pacientes posoperados inmediatos portadores de una sonda vesical (neurológicos, ginecológicos y urológicos).

Se utilizó una guía de recolección de datos, tomándose en cuenta 9 dimensiones que a su vez se subdividen en: datos generales como la edad (mayores y menores de 40 años) y el género (femenino 
y masculino); seguido de los datos específicos: antecedentes de enfermedades (neuropatía diabética, hiperactividad del detrusor con contractilidad alterada, adenoma prostático, retención urinaria psicógena e infecciones urinarias); tipo de cirugía (cirugía general, traumatología, otorrino, cabeza y cuello, cirugía oncológica, tórax y cardiovascular); tiempo operatorio (mayor y menor de 2 horas); tipo de anestesia (epidural, raquídea y general); uso de narcóticos (petidina y fentanilo); dosis de narcóticos administrado (petidina: menor o mayor de 50mg, fentanilo: menor o mayor a $100 \mathrm{ug}$ ), volumen transfundido (menor o mayor a 2 litros), y la presencia de globo vesical (sí o no). Este instrumento fue validado por juicio de expertos. Cabe recalcar que para la confiabilidad del instrumento no se utilizó un estadístico debido a que el estudio fue de casos y controles. Se seleccionó una muestra que la conformó fueron los pacientes que presentaron retención urinaria; a los cuales se les denominó casos. De la misma manera, a los pacientes que no presentaron retención urinaria los cuales fueron el grupo control, de esta manera se logró medir las variables para la identificación de factores de riesgo.
La guía de observación elaborada ayudó a ver retrospectivamente cuáles fueron los factores que influyeron en el desarrollo de la retención urinaria. Y de esta manera se hizo una comparación entre ambos grupos.

Los datos se obtuvieron en un solo momento, incluyendo las características generales de los pacientes. Una vez obtenidos los datos, se identificaron los factores de riesgo, y el cálculo se realizó usando el estadístico de Odds Ratio (OR): Medida de asociación entre un factor de riesgo y la enfermedad que resulta del cociente entre el Odds del grupo expuesto sobre el Odds del grupo no expuesto. Expresa cuántas veces más probable es el riesgo de contraer la enfermedad en presencia de un factor de exposición que en su ausencia con su respectivo intervalo de confianza (IC) y significancia al 95\%.

Se procedió a la tabulación, presentación en tablas descriptivas e inferenciales, con el empleo de programas y software; Office vol. 2007, SPSS vol.19.

\section{RESULTADOS}

Tabla 1

Distribución de pacientes posoperados inmediatos según género de la Clínica Good Hope, 2012.

\begin{tabular}{lcc}
\hline Género & N. ${ }^{\circ}$ & $\%$ \\
\hline Femenino & 44 & 51.8 \\
Masculino & 41 & 48.2 \\
\hline Total & 85 & 100
\end{tabular}

En la tabla 1 se muestra que el $51,8 \%$ (44) de género masculino el 48,2\% (41). pacientes corresponden al género femenino, y al

Tabla 2

Distribución de pacientes posoperados inmediatos según edad de la Clínica Good Hope, 2012.

\begin{tabular}{lcc}
\hline Edad & N. ${ }^{\circ}$ & $\%$ \\
\hline Menor de 40 años & 35 & 41.2 \\
Mayor de 40 años & 50 & 58.8 \\
\hline Total & 85 & 100
\end{tabular}

La tabla 2 muestra que más de la mitad $(58,8 \%)$ 40 años. tuvieron más de 40 años y el 41,2\% tienen menos de 
Tabla 3

Factores de riesgo que influyen en la retención urinaria en los pacientes posoperados inmediatos de la Clínica Good Hope, abril 2012.

\begin{tabular}{|c|c|c|c|}
\hline \multirow[t]{2}{*}{ Factor } & \multirow{2}{*}{$\frac{\text { Valor Sigma }}{\text { OR }}$} & \multicolumn{2}{|c|}{ Desviación Estándar } \\
\hline & & Inferior & Superior \\
\hline Edad & .433 & .173 & 1.087 \\
\hline Género & .399 & .164 & .974 \\
\hline \multicolumn{4}{|l|}{ Enfermedades } \\
\hline Hiperactividad del detrusor & 3.125 & .272 & 35.893 \\
\hline Adenoma de próstata & $11.692 *$ & 1.302 & 105.028 \\
\hline Infecciones urinarias & 3.125 & .272 & 35.893 \\
\hline \multicolumn{4}{|l|}{ Especialidades } \\
\hline Cirugía general & .924 & .387 & 2.207 \\
\hline Traumatología & 4.400 & 1.366 & 14.172 \\
\hline Otorrinolaringología & .190 & .022 & 1.624 \\
\hline Cirugía oncológica & .485 & .048 & 4.866 \\
\hline Cirugía de tórax y cardiovascular & .227 & .026 & 1.979 \\
\hline Duración de la cirugía & .668 & .277 & 1.612 \\
\hline \multicolumn{4}{|l|}{ Tipo de anestesia } \\
\hline Epidural & 3.587 & 1.177 & 10.932 \\
\hline Raquídea & 11.717 & 2.399 & 57.229 \\
\hline General & .117 & .043 & .320 \\
\hline \multicolumn{4}{|l|}{ Tipo de opioides } \\
\hline Petidina & .452 & .113 & 1.807 \\
\hline Fentanilo & 1.091 & .438 & 2.718 \\
\hline Petidina y fentanilo & .707 & .218 & 2.287 \\
\hline \multicolumn{4}{|l|}{ Dosis de opioides } \\
\hline Fentanilo & .888 & .371 & 2.124 \\
\hline Volumen transfundido & .759 & .317 & 1.813 \\
\hline
\end{tabular}

En la tabla 3, respecto a los factores de riesgo que influyen en la retención urinaria de los pacientes posoperados inmediatos de la Clínica Good Hope, abril 2012 se observa que la edad mayor o menor de 40 años no representó un factor de riesgo $(\mathrm{OR}=0,433)$ que influya en la retención urinaria de pacientes posoperados, asimismo, el ser de género masculino o femenino $(\mathrm{OR}=0,399)$.

Al tener antecedentes patológicos o enfermedades en los pacientes posoperados, se encontró que los pacientes con antecedentes de enfermedades prostáticas obtuvieron un $(\mathrm{OR}=11,692 ; \mathrm{I}=1,302$ y $\mathrm{S}=105,028)$, es decir 11,6 veces más posibilidades de presentar retención urinaria respecto a los otros pacientes con antecedentes patológicos diversos consignados en el estudio, lo que indica que existe una alta significancia en el estudio.
Por otro lado, el tipo de cirugía o a la especialidad por la que fueron operados los pacientes, se halló que los intervenidos por traumatología representa $(\mathrm{OR}=4,4$; $\mathrm{I}=1,366$ y $\mathrm{S}=14,172$ ) 4,4 veces más posibilidades de presentar retención urinaria como factor de riesgo en el estado postoperatorio inmediato, frente a las otras especialidades a los que fueron sometidos, dichos pacientes.

Los pacientes posoperados sometidos a anestesia raquídea tuvieron 11,7 veces más posibilidad $(\mathrm{OR}=11,717 ; \mathrm{I}=2,399$ y $\mathrm{S}=57,229)$ de padecer retención urinaria en el estado posoperatorio inmediato, respecto a los pacientes sometidos a anestesia general. Lo que demuestra que la anestesia raquídea está altamente asociada a desarrollar retención urinaria posoperatoria. Del mismo modo, se halló que los pacientes sometidos a anestesia epidural tuvieron un 
mediano riesgo de $(\mathrm{OR}=3,587 ; \mathrm{I}=1,177$ y $\mathrm{S}=10,932)$ 3,5 veces más posibilidad de padecer retención urinaria en el posoperatorio inmediato, en relación a otros tipos de anestesia.

\section{DISCUSIÓN}

El someter a un paciente a intervenciones quirúrgicas, implica riesgos derivados de la anestesia y la cirugía misma. Las complicaciones o riesgos anestésicos más comunes ya han sido identificados en pacientes sometidos a cirugía general. Dentro de este conjunto de complicaciones derivadas de la anestesia espinal, una es, la retención urinaria (Dálbora, y otros, 2008).

La retención urinaria, tras anestesia y cirugía, tiene una incidencia muy significativa que conllevan a tener en consideración fines terapéuticos y preventivos en pacientes posoperados. Esta retención urinaria posoperatoria se define como la incapacidad para miccionar en presencia de una vejiga repleta (Rodríguez, Vásquez, Martínez, \& Carballido, 2011).

Considerando el objetivo del estudio, de identificar los factores de riesgo que influyen en la retención urinaria en los pacientes posoperados inmediatos y los hallazgos, estos no expresaron diferencias significativas $(\mathrm{OR}=0,433$ y $\mathrm{OR}=0,399)$ respectivamente, pues no influyen en la retención urinaria posoperatorias. Este resultado concuerda con otros estudios de Liang, Chang, Wong, \& Cheng, (2010), quienes hallaron que la edad menor de 40 o mayor de 60 años, los del sexo femenino fueron factores predisponentes para la retención urinaria.

Sin embargo, Bueno Lledó (2002) afirma “...que la edad y el género no deben ser factores excluyentes para producir complicaciones posoperatorias, como la retención urinaria...". También para Barretto de Carvalho, Vieira da Costa, \& Saraiva (2007), la edad y el género no constituyen factores de riesgo en la producción de complicaciones posoperatorias, incluyendo la retención urinaria. Haciendo entrever que aún prevalecen ciertas discrepancias respecto al género y edad como factores de riesgo.

Se pudo observar que en los pacientes de género masculino y con antecedentes de adenoma de próstata tuvieron un factor de riesgo $(\mathrm{OR}=11,692)$ de 11,6 veces razones de ventajas en la retención urinaria respecto a los otros pacientes con antecedentes patológicos diversos. Estos datos muestran similitud con lo encontrado por Chung (2007), quien manifiesta: “... el ser anciano, la enfermedad pulmonar, el cáncer rectal, el tiempo operatorio prolongado y el procedimiento pélvico adicional fueron los factores de riesgo de disfunción urinaria,...”. Para Verger-Kuhnke, Reuter, Epple, Ungemach, \& Beccaría (2007), en las cirugías de adenomas prostáticos, las complicaciones posoperatorias severas son generalmente hematurias, náuseas, dolor y retención urinaria con mayor frecuencia. También se halló que en pacientes intervenidos por traumatología tuvieron $(\mathrm{OR}=4,4)$ 4,4 veces más posibilidades de desarrollar retención urinaria durante el postoperatorio inmediato, frente a las otras especialidades de intervención. Existen autores que apoyan el presente estudio, mencionando que los procesos quirúrgicos, como el de traumatología, suelen tener complicaciones como sangrado, dolor agudo intenso y retención urinaria de forma continua.

Se identificó que el empleo de la anestesia raquídea, tuvo $(\mathrm{OR}=11,717) \quad 11.7$ veces más posibilidades de presentar retención urinaria, respecto a los pacientes sometidos a anestesia general; los que recibieron anestesia epidural tuvieron $(\mathrm{OR}=3,587) 3,5$ veces de mediana posibilidad de padecer retención urinaria. Estos hallazgos son parecidos al trabajo de Jensen (1991), quien encontró una incidencia significativamente menor de retención urinaria con anestesia local, comparado con la anestesia general y regional.

Darrah, Griebling, \& Silverstein (2009) manifiestan que el riesgo de retención urinaria es elevado después de la cirugía anorrectal de la herniorrafia, utilizando anestesia espinal con anestésicos locales de acción prolongada y la analgesia epidural. El sondaje se recomienda cuando el volumen de la vejiga excede los $600 \mathrm{ml}$, para evitar las secuelas negativas de la sobredistensión prolongada de la vejiga.

\section{CONCLUSIONES}

Finalmente, se pudo identificar diversos factores asociados a la retención urinaria en los pacientes posoperatorios inmediatos, concluyendo que los pacientes intervenidos bajo anestesia raquídea tienen mayor probabilidad de desarrollar retención urinaria. Por consiguiente, el adquirir conocimientos acerca de estos factores de riesgo permitirá anticipar, planificar y controlar las posibles complicaciones y, de este modo, brindar cuidados de calidad de carácter humanístico y de un enfoque científico a los pacientes. 


\section{REFERENCIAS BIBLIOGRÁFICAS}

Barretto de Carvalho, M., Vieira da Costa, V., \& Saraiva, R. (marzo-abril de 2007). Retención urinaria posoperatoria: evaluación de pacientes en tratamiento analgésico con opioides. Latino-am Enfermagen, 15(2).

Bueno Lledó J, Planeéis Roig M, Arnau Bertomeu C, et al. Colecistectomía laparoscópica ambulatoria. ¿El nuevo "gold standard" de la colecistectomía?. Rev. Es Enferm. Dig. 2006.

Chung, C. (Octubre de 2007). Cirugía coloproctológica. (T. Departamento de Cirugía Colorrectal y Urología. Chang Gung Memorial Hospital. Colegio Universitarios de Tipei, Productor) Recuperado el Diciembre de 2012, de cirugía Coloproctológica.

Dálbora, J., Fuentes, C., Miño, M., Bravo, T., Vicencio, M., \& Madrid, E. (2008). Factores de riesgo de retención urinaria aguda en cirugía mayor ambulatoria bajo anestesia espinal. Chilena de Cirugía, 60(2), 139-144.

Darrah, D., Griebling, T., \& Silverstein, J. (Setiembre, 2009). Postoperative Urinary Retention. Journal of Anaesthesiology Clinic, 27(3), 465-484.

Hamlin, L. (2010). Enfermería perioperatoria: texto introductorio. México: Editorial El Manual Moderno.
Jensen, V. (1991). The Trup Syndrome. Canadian Journal of Anesthesia, 38(1), 90-97.

Liang, C., Chang, D., Wong, Y., \& Cheng, J. (Octubre de 2010). Efectos de la analgesia postoperatoria en retenciónurinaria postparto en mujeres sometidas a cesárea. (E. D. Taiwan, Ed.) journalof Obstetrics and Gyneacology Research, 36(5), 91-95.

Morgan, E., Murray, M., \& Mikhail, M. у. (2003). Anestesiología clínica (Tercera ed.). México: Editorial El Manual Moderno.

Océano/Centrum. (1997). Manual de enfermería. España: Océano.

Rodríguez, G., Vásquez, D., Martínez, J., \& Carballido, J. (2011). Hiperplasi benigna de próstata. Medicine, $10(83)$.

Smeltzer, S., \& Bare, B. (2005). Enfermería medicoquirúrgico (Décima ed.). México: McGraw Hill Interamericana.

Verger-Kuhnke, A., Reuter, M., Epple, W., Ungemach, G., \& Beccaría, M. (2007). La vaporización fotoselectiva del adenoma prostático con el KTP-láser (Kaliumtitanyl-phosphat) de 80 watt y la RTU-P de baja presión hidráulica. (A. Servicio de Urología del Hospital Karl olga. Hospital Escuela de la Universidad de Ulm, Ed.) Chilena de Urilogía, 72(3), 238-249. 